Original paper

\title{
Fiducial marker matching versus vertebral body matching: Dosimetric impact of patient positioning in carbon ion radiotherapy for primary hepatic cancer
}

\author{
Satoshi Abe ${ }^{\mathrm{a}}$, Yoshiki Kubota ${ }^{\mathrm{b}, *}$, Kei Shibuya ${ }^{\mathrm{c}}$, Yoshinori Koyama ${ }^{\mathrm{c}}$, Takanori Abe ${ }^{\mathrm{c}}$, Tatsuya Ohno ${ }^{\mathrm{b}}$, \\ Takashi Nakano ${ }^{\mathrm{b}, \mathrm{c}}$ \\ ${ }^{a}$ Department of Radiology, Gunma University Hospital, 3-39-15 Showa-machi, Maebashi, Gunma 371-8511, Japan \\ ${ }^{\mathrm{b}}$ Gunma University Heavy Ion Medical Center, 3-39-22 Showa-machi, Maebashi, Gunma 371-8511, Japan \\ ${ }^{\mathrm{c}}$ Department of Radiation Oncology, Gunma University Graduate School of Medicine, 3-39-22 Showa-machi, Maebashi, Gunma 371-8511, Japan
}

\section{A R T I C L E I N F O}

\section{Article history:}

Received 22 July 2016

Received in Revised form 22 December 2016

Accepted 27 December 2016

Available online 3 January 2017

\section{Keywords:}

Carbon ion radiotherapy

Hepatic cancer

Fiducial marker matching

Vertebral body matching

\begin{abstract}
A B S T R A C T
Purpose: The aim of this study was to compare the dose-volume parameters of fiducial marker matching (MM) with vertebral body matching (VM) in patient positioning for carbon ion radiotherapy for primary hepatic cancer.

Materials and methods: Twenty patients with primary hepatic cancer were retrospectively studied to assess changes in reproducibility of tumor position and dose distribution on two CT scans. One was for treatment planning and another was for dose confirmation, acquired the day before the first treatment day. The coverage of the clinical target volume (CTV) $\left(D_{98}\right)$ and normal liver volume excluding the CTV which received $20 \mathrm{~Gy}$ relative biological effectiveness (RBE) $\left(V_{20}\right)$ were used as evaluation parameters. Additionally, the correlation of tumor movement and $\mathrm{D}_{98}$ was calculated in VM and MM. The prescription dose was $60.0 \mathrm{~Gy}$ (RBE) delivered in four fractions ( $15 \mathrm{~Gy} / \mathrm{fx})$.

Results: The median (range) $\mathrm{D}_{98}$ for VM and MM was 57.9 (20.8-59.9) and 59.9 (57.2-60.3) Gy (RBE), respectively. The median (range) $\mathrm{V}_{20}$ for $\mathrm{VM}$ and $\mathrm{MM}$ was 17.9 (4.8-44.4) and 16.2 (4.7-44.9) Gy (RBE), respectively. The $\mathrm{D}_{98}$ for MM was significantly larger than that for VM $(p=0.001)$, although $\mathrm{V}_{20}$ showed no significant difference $(p>0.05)$. Twelve patients were clinically acceptable $\left(D_{98}>57 \mathrm{~Gy}\right.$ (RBE)) with VM, while all patients were clinically acceptable with MM. Marker movement correlated with a decrease of $\mathrm{D}_{98}$ for VM $(\mathrm{R}=-0.814)$.

Conclusion: Compared with VM, MM was clinically acceptable in all patients. This suggests that MM is more robust than VM.

(C) 2016 Associazione Italiana di Fisica Medica. Published by Elsevier Ltd. This is an open access article under the CC BY-NC-ND license (http://creativecommons.org/licenses/by-nc-nd/4.0/).
\end{abstract}

\section{Introduction}

Particle beams, such as proton and carbon ion beams, are characterized by a steep dose distribution (Bragg peak) at the end of a range [1-3]. Using the concept of a spread-out Bragg peak (SOBP), carbon ion beams provide excellent dose conformity to the target region. However, unless the water-equivalent path length (WEPL) to the target is determined and accounted for, the position of the Bragg peak may differ from what is planned and the target coverage could be insufficient [4]. Additionally, because of the steeper lateral penumbra of carbon ion beams compared with X-rays or proton beams, dose coverage for the target region is sensitive to organ motion perpendicular to the beam path [5]. In the conven-

\footnotetext{
* Corresponding author.

E-mail address: y_kubota@gunma-u.ac.jp (Y. Kubota).
}

tional broad-beam delivery system with carbon ions for abdominal cancers, bony structures such as the pelvis and vertebral bodies, which have large stopping powers, have been used for daily position verification. However, even when the bony structure is matched, if internal anatomical changes occur along the beam path during fractionated carbon ion radiotherapy (CIRT), the planned dose may not be reproduced [6-10].

In CIRT for primary hepatic cancer, our institution uses two types of markers as a reference to the target position. One is a metal marker inserted at the peripheral liver tissue near the target under computed tomography (CT) guidance [11,12], and the other is a residual lipiodol delivered via transcatheter arterial chemoembolization [13]. These markers act as fiducial markers for patient positioning. Fiducial marker matching (MM) and tracking systems are currently available for X-ray radiotherapy [14-18]. However, the clinical significance of MM in patient positioning for CIRT has 
not been fully investigated. Therefore, we verified the effectiveness of MM for patients with primary hepatic cancer by comparing the dose-volume parameters of vertebral body matching (VM) with that of MM.

\section{Materials and methods}

\subsection{Patients}

CT images for 23 consecutive patients treated at our facilities from November 2011 and August 2013 were acquired. Three patients were excluded from the analysis because their CT images were not continuous due to unstable breathing patterns. Data from 20 patients were retrospectively studied with respect to changes in dose-volume parameters. Patient characteristics are shown in Table 1 . This study was approved by the Institutional Review Board at Gunma University Hospital (approval number: 15-88). Consent to participate was not required owing to the retrospective nature of the study and anonymity of the data.

\subsection{Patient immobilization}

The patients were immobilized by fixation with a shell fitter $(600 \times 800 \mathrm{~mm}$; Kuraray, Tokyo, Japan) for abdominal suppression and prevention of body movement, Moldcare $(600 \times 600 \mathrm{~mm}$; Alcare, Tokyo, Japan) for trunk support, and a vacuum cushion (Esform; Engineering System, Matsumoto, Japan) for leg stabilization. The patients were immobilized in either the supine or prone position depending on factors such as the target position and organ (s) at risk (OAR).

\subsection{Treatment planning $C T$}

A CT scanner (Aquilion LB; Toshiba Medical Systems, Tochigi, Japan) was used to acquire images for treatment planning. The CT gantry is self-propelled and the couch has six axes with a translating $(x, y, z)$ and rotating $\left(\theta_{x}, \theta_{y}, \theta_{z}\right)$ drive mechanism. A respiratory-gated $\mathrm{CT}$ image set was acquired for treatment planning (PlanCT) after immobilization [19]. Respiration was monitored with a respiratory gating system (AZ-733 V with laser respiration sensor; Anzai Medical, Tokyo, Japan) [20]. PlanCT images were acquired near the point of maximum expiration while breathing freely. After obtaining the PlanCT, the respiratory movement of the fiducial marker was measured on the four-dimensional (4D)CT images [21-23]. The 4DCT projection data were acquired using a helical scan mode and the 4DCT images were reconstructed using a phase-based respiratory binning method. The 4DCT images might include motion artifacts in a time resolution of $0.3 \mathrm{~s}$ (time for half reconstruction) around the phases. The 4DCT images were reconstructed from five phase images. Here, the maximum expiration phase was defined as $0 \%$, and the highest inspiration phase was defined as $100 \%$ for the respiratory waveform. The 4DCT image was reconstructed to $30 \%, 10 \%$ for both expiration and inspiration phases, and $0 \%$. The motion surrogate of the tumor was defined as the motion of the fiducial marker. The 4DCT images were also used to confirm the dose distribution during respiratory gating. Contrast-enhanced CT images in the early and delayed phases were then acquired for contouring and margin definition of the tumor during exhalation.

\subsection{Treatment planning}

XiO-N (Mitsubishi Electric, Tokyo, Japan) planning software, which incorporates a dose engine for ion beam radiotherapy dose calculations (K2-Dose) [24-27], was used for treatment planning. After a radiation oncologist contoured the gross tumor volume (GTV), the clinical target volume (CTV) was created from the GTV with a 5-mm margin. The planning target volume (PTV) was then created from the CTV to achieve the total margin (TM). The TM was calculated as the square root of the sum of the squared setup margin and squared internal margin. The PTV was adjusted when an OAR, such as the main portal vein, was present near the target. Here, the CT images on which marker movement was greater for two $30 \%$ phase images reconstructed from 4DCT were used to calculate the internal margin. Basically, the treatment was performed between the $30 \%$ expiration phase and the $30 \%$ inspiration phase. However, if the margin resulted in excessive dose to the OAR, the $10 \%$ expiration phase or the $10 \%$ inspiration phase was used to calculate the internal margin. In all cases, the setup margin was established to be precisely $3 \mathrm{~mm}$ [21]. The treatment plans were generated to deliver 95\% of the prescription dose in the PTV.

A range compensator (bolus) was used to adjust the range, and the bolus smearing margin ranged from 3 to $7 \mathrm{~mm}$. This was determined from the TM. One horizontal beam and one vertical beam

Table 1

Patient demographics.

\begin{tabular}{|c|c|c|c|c|c|c|c|}
\hline Patient & Sex & Age & Tumor location & Tumor volume (ml) & Patient position & Irradiation direction & Matching object \\
\hline 1 & $\mathrm{~F}$ & 82 & S6 & 3.2 & PR & RL, PA & Metal \\
\hline 2 & $\mathrm{~F}$ & 71 & S7 & 26.9 & PR & RL, PA & Metal \\
\hline 3 & $\mathrm{~F}$ & 71 & S7 & 11.2 & PR & RL, PA & Lipiodol \\
\hline 4 & M & 83 & S6 & 53.4 & PR & RL, PA & Metal \\
\hline 5 & M & 65 & S8 & 110.0 & $\mathrm{SP}$ & $\mathrm{RL}, \mathrm{AP}$ & Metal \\
\hline 6 & M & 80 & S8 & 36.1 & SP & RL, AP & Metal \\
\hline 7 & M & 63 & S8 & 65.8 & SP & $\mathrm{RL}, \mathrm{AP}$ & Lipiodol \\
\hline 8 & M & 57 & S8 & 5.2 & SP & RL, AP & Lipiodol \\
\hline 9 & M & 80 & S4 & 23.0 & SP & $\mathrm{RL}, \mathrm{AP}$ & Lipiodol \\
\hline 10 & M & 76 & S8 & 56.9 & PR & RL, PA & Lipiodol \\
\hline 11 & $\mathrm{~F}$ & 75 & S5 & 46.5 & PR & RL, PA & Lipiodol \\
\hline 12 & M & 79 & S3 & 142.4 & SP & RL, AP & Metal \\
\hline 13 & M & 72 & S3 & 3.1 & SP & LR, AP & Metal \\
\hline 14 & M & 83 & S5/6 & 46.3 & SP & $\mathrm{RL}, \mathrm{AP}$ & Metal \\
\hline 15 & M & 73 & S8 & 141.9 & PR & RL, PA & Lipiodol \\
\hline 16 & M & 75 & S3 & 10.3 & $\mathrm{SP}$ & LR, AP & Metal \\
\hline 17 & M & 81 & S8 & 53.2 & SP & RL, AP & Metal \\
\hline 18 & $\mathrm{~F}$ & 73 & S5 & 14.0 & SP & RL, AP & Metal \\
\hline 19 & $\mathrm{~F}$ & 74 & S5 & 33.6 & PR & RL, PA & Metal \\
\hline 20 & $\mathrm{~F}$ & 81 & S8 & 12.5 & $\mathrm{SP}$ & $\mathrm{RL}, \mathrm{AP}$ & Metal \\
\hline
\end{tabular}

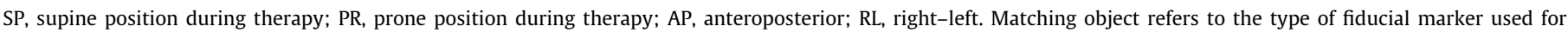
position matching. 
were used in the treatment plans for all patients. The prescription dose was set at $60 \mathrm{~Gy}$ relative biological effectiveness (RBE) delivered in four fractions based on an established protocol, and each beam was generated using a SOBP [28] with a passive irradiation method [29]. Representative treatment planning images are shown in Fig. 1.

\subsection{Pre-treatment verification}

Pre-treatment verification was performed in the CT simulation room before the first day of therapy. Orthogonal X-ray and CT images were obtained. The couch allows for movement of the patient from the X-ray system to the CT scanner without any repositioning. The patients were immobilized, and position matching with the orthogonal X-ray images was performed to confirm the reproducibility of the treatment position. Respiratory-gated images were then acquired to confirm the reproducibility of the internal position by the same method that was used for the PlanCT scan. This image set was defined as ConfCT. The ConfCT images were also used to confirm the dose distribution and subsequent analysis.

\subsection{Evaluation methods}

The PlanCT and ConfCT images were manually registered in the Focal Contouring System (Elekta, Stockholm, Sweden) based on translational degrees of freedom exclusively along the $x$ (leftright), $y$ (cranial-caudal), and $z$ (abdominal-dorsal) axes without rotations. If a twist existed between the upper vertebral body and the lower vertebral body when the PlanCT and ConfCT images were registered on the vertebral body near the tumor with the translations, the position of the vertebral body near the tumor was adjusted to the center region of the twist. In the MM, two images were registered with the fiducial marker position from
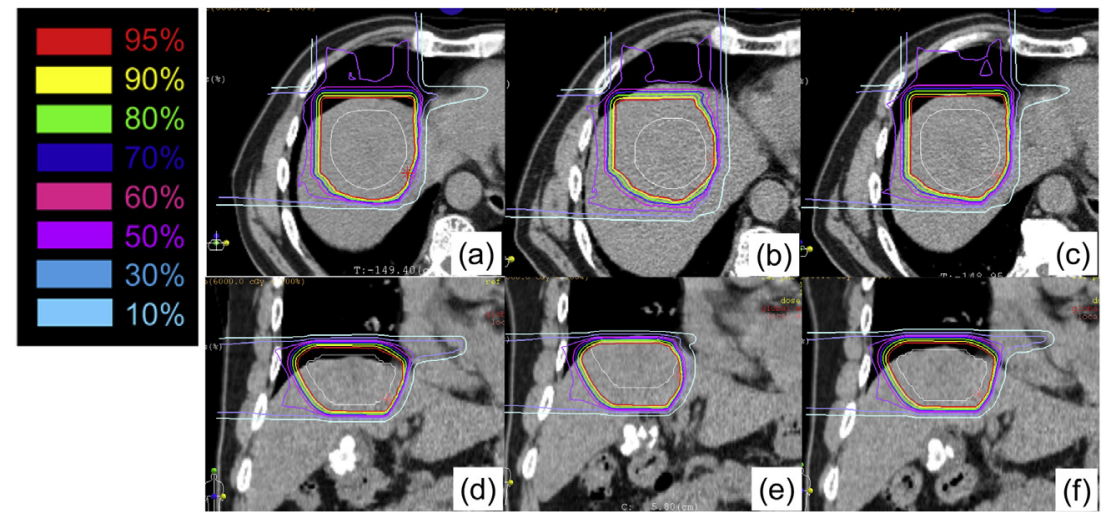

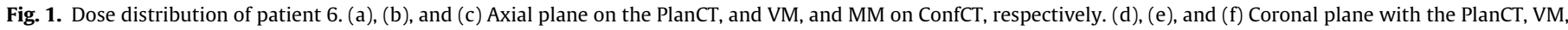
and MM, respectively, on ConfCT. The CTV is outlined in white. The dose distribution is displayed on a graduated scale from $10 \%$ to $95 \%$.
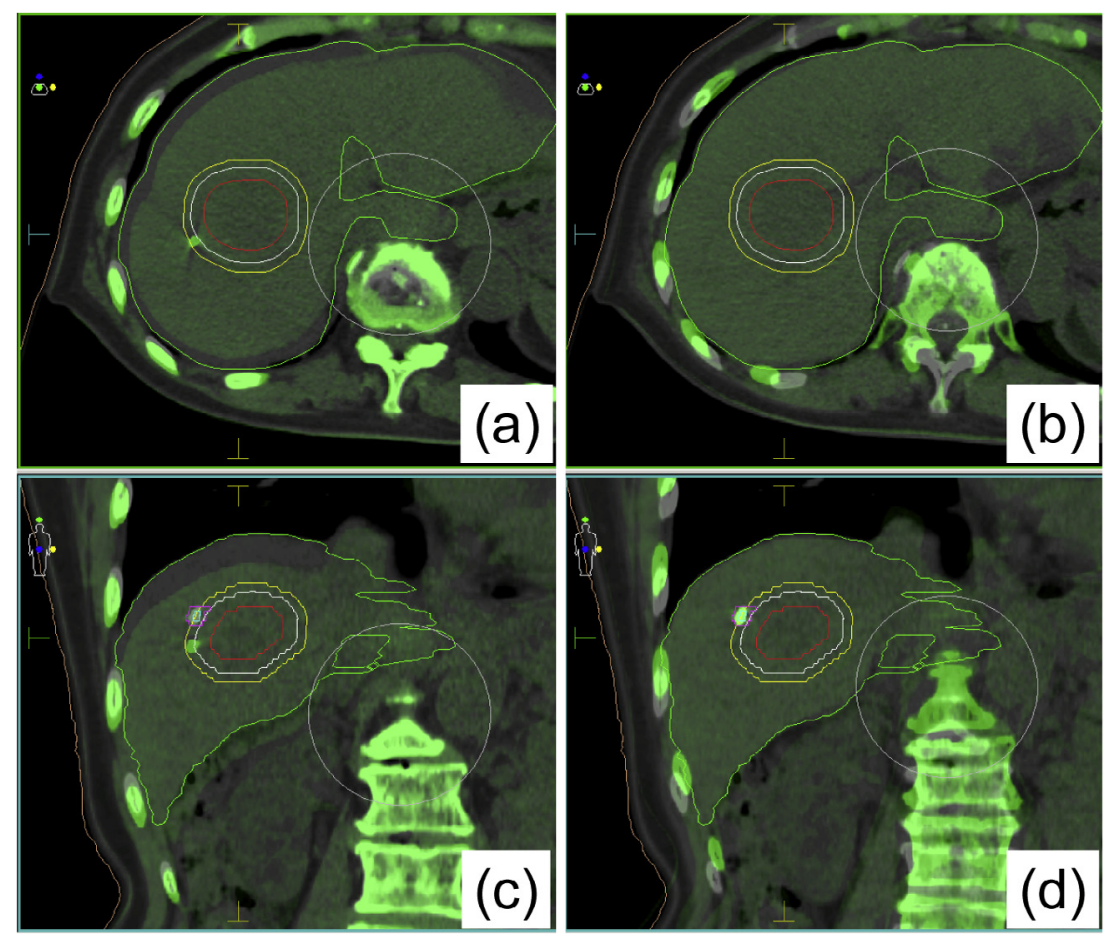

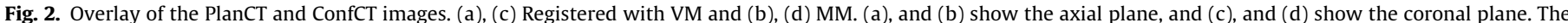

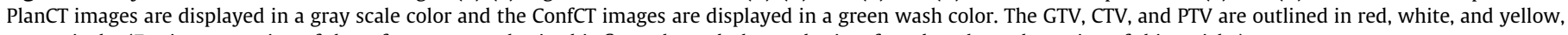
respectively. (For interpretation of the references to color in this figure legend, the reader is referred to the web version of this article.) 


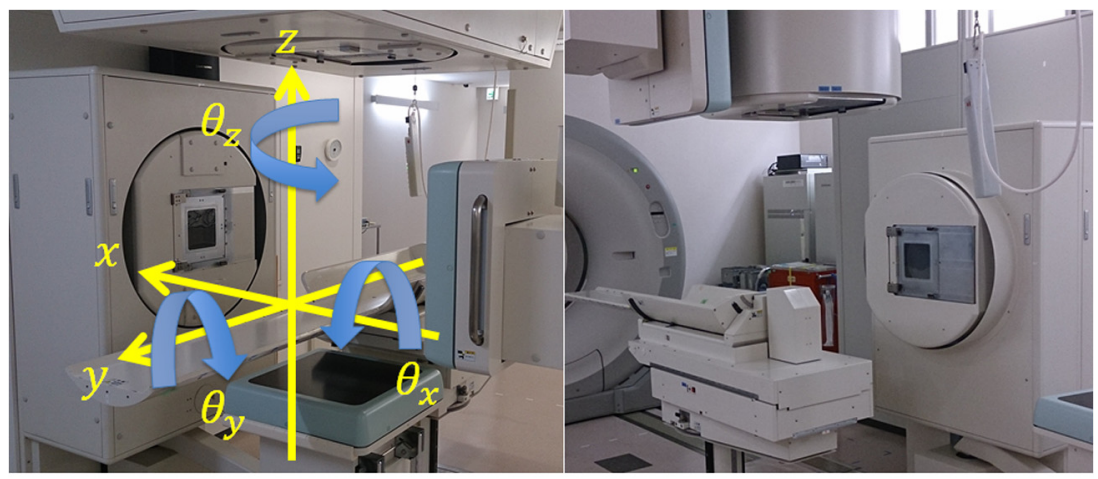

(a) treatment room

(b) CT simulation room

Fig. 3. Photograph of treatment room (a) and CT simulation room (b). The patient direction was defined as the direction shown in the photograph.

the position determined by the VM position on translation only without rotation. The rigid registration method was used for all registrations. A representative image of each registration method is shown in Fig. 2. The VM and MM registering methods were then applied, and each beam isocenter was set for dose calculation on the ConfCT images. The rotation angle was not corrected for because image rotation is impossible with the XiO-N system and any rotations on the Focal Contouring System are not reflected to the XiO-N. The fiducial marker movement was measured from the VM position to the MM position. On these axes, left is + , right is -, cranial is + , caudal is -, abdominal is + , and dorsal is - (Fig. 3 ).

Using XiO-N, the ConfCT was used to recalculate the dose at isocenter as determined on the Focal Contouring System with the beam parameters from the original treatment plan. The minimum dose required to cover $98 \%$ of the CTV $\left(D_{98}\right)$, the volume which received over $80 \%, 90 \%, 95 \%$ of prescribed dose in the $C T V\left(V_{80}\right.$, $\mathrm{V}_{90}, \mathrm{~V}_{95}$ ), and normal liver volume, excluding the CTV, which received $20 \mathrm{~Gy}$ ( $\mathrm{RBE})\left(\mathrm{V}_{20}\right)$, were used to evaluate the dose distribution from the dose-volume histogram (DVH). In addition, the clinically acceptance tolerance of $\mathrm{D}_{98}$ was defined as $57 \mathrm{~Gy}$ (RBE) (95\% of prescribed dose) on the ConfCT.

For each matching method, the $\mathrm{D}_{98}$ and $\Delta D$, defined in $\mathrm{Eq}(1)$, were used to evaluate the relationship between the target coverage and target movement to the TM.

$\Delta D=\sqrt{M_{x}^{2}+M_{y}^{2}+M_{z}^{2}}-\sqrt{T M_{x}^{2}+T M_{y}^{2}+T M_{z}^{2}}$,

where $M_{x, y, z}$ is defined as the fiducial marker movement between the PlanCT and ConfCT on the $x, y$ and $z$ axes and $T M_{x, y, z}$ is defined as the TM of the $M_{x, y, z}$ direction on the $x, y$ and $z$ axes.

Statistically significant differences in each dose volume parameter for each matching method were analyzed by the Friedman test (with Holm corrections for multiple comparisons) with statistical analysis software (EZR version 1.33) [30]. The level of statistical significance was set at $5 \%$.

In addition, the magnitude of the change in WEPL from the beam isocenter to the skin surface on the PlanCT and ConfCT with the MM were measured for all beams to analyze the WEPL change along the beam line.

\section{Results}

\subsection{Relationship between $\Delta D$ and $D_{98}$}

The median (range) inter-fractional error of the fiducial marker between the PlanCT and ConfCT in the $x, y$, and $z$ directions was -0.5 ( -3.9 to 5.8 ), 3.4 ( -11.5 to 12.2 ), and -0.3 ( -6.8 to 8.6 )

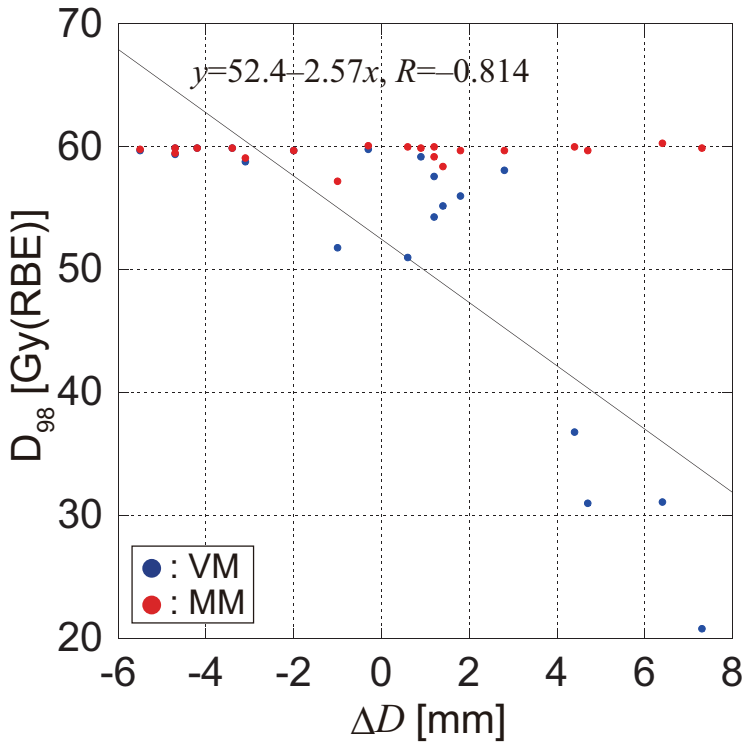

Fig. 4. The relationship between $D_{98}$ and $\Delta D$. The vertical axis shows the $D_{98} G y$ (RBE), and the horizontal axis shows the $\Delta D(\mathrm{~mm})$. The red point indicates MM. The blue point indicates VM. The correlation coefficient was -0.814 on the VM, while that could not be defined on the MM because the degree of inclination and the dispersion were almost 0 . The blue area is $\Delta D>-1.8 \mathrm{~mm}$. (For interpretation of the references to color in this figure legend, the reader is referred to the web version of this article.)

$\mathrm{mm}$, respectively. The relationship between $\Delta D$ and the $\mathrm{D}_{98}$ for each matching method is shown in Fig. 4. The correlation coefficient was -0.814 with VM, though it could not be defined with MM because the gradient and the standard deviation were almost 0 . Furthermore, the $D_{98}$ of all patients with MM was clinically acceptable. In contrast, the $\mathrm{D}_{98}$ for 12 out of the 20 patients with VM was clinically acceptable. Using a linear approximation for $\Delta D$ and $D_{98}$, the acceptance criteria for VM was satisfied when $\Delta D$ was less than $-1.8 \mathrm{~mm}$.

\subsection{Dose volume parameters for two different matching methods}

The median (range) $\mathrm{D}_{98}$ for $\mathrm{VM}, \mathrm{MM}$, and original treatment plan was 57.9 (20.8-59.9), 59.9 (57.2-60.3), and 59.8 (59.1-60.1) $\mathrm{Gy}(\mathrm{RBE})$, respectively. The $\mathrm{D}_{98}$ of MM was significantly larger than that of VM $(p=0.001)$. Moreover, the $\mathrm{D}_{98}$ of the original treatment plan was significantly larger than that of VM $(p=0.001)$ while no significant difference was observed for MM $(p>0.05)$. The median 
Table 2

Dose volume parameters for vertebral body matching and fiducial marker matching.

\begin{tabular}{|c|c|c|c|c|}
\hline Object & Coverage & Plan [median (range)] & VM [median (range)] & MM [median (range)] \\
\hline CTV & $\begin{array}{l}\mathrm{V}_{80}[\%] \\
\mathrm{V}_{90}[\%] \\
\mathrm{V}_{95}[\%] \\
\mathrm{D}_{98}[\mathrm{~Gy}(\mathrm{RBE})]\end{array}$ & $\begin{array}{l}100(100-100) \\
100(99.7-100) \\
100(99.2-100) \\
59.8(59.1-60.1)\end{array}$ & $\begin{array}{l}100(89.6-100) \\
99.7(83-100) \\
100(78-100) \\
57.9(20.8-59.9)\end{array}$ & $\begin{array}{l}100(99.8-100)^{* *} \\
100(99.1-100)^{* *} \\
100(98.1-100)^{*} \\
59.8(57.2-60.3)^{* * *}\end{array}$ \\
\hline Liver-CTV & $\mathrm{V}_{20}[\mathrm{~Gy}(\mathrm{RBE})]$ & $16.8(5.1-45.9)$ & $17.9(4.8-44.4)$ & $16.2(4.7-44.9)$ \\
\hline
\end{tabular}

${ }^{*} \mathrm{p}<0.05$ compared with the plan.

** $\mathrm{p}<0.05$ compared with VM.

(a)

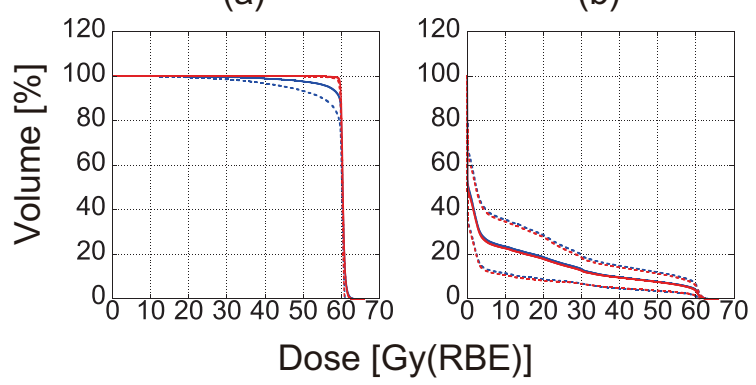

Fig. 5. Averaged DVH for 20 patients for plan recalculated on registered ConfCT. (a) CTV, (b) normal liver. The vertical axis shows the relative volume (\%), and the horizontal axis shows the dose Gy (RBE). The blue line indicates VM. The red line indicates MM. The dashed lines indicate subtraction of one standard deviation in (a) and subtraction or addition of one standard deviation in (b). (For interpretation of the references to color in this figure legend, the reader is referred to the web version of this article.)

(range) of $\mathrm{V}_{20}$ of the normal liver for $\mathrm{VM}, \mathrm{MM}$, and original treatment plan was 17.9 (4.8-44.4), 16.2 (4.7-44.9), and 16.8 (5.145.9) Gy (RBE), respectively. There was no significant difference in the $\mathrm{V}_{20}$ between $\mathrm{VM}$ and $\mathrm{MM}$ and the original treatment plan $(p>0.05)$. The results for each index are shown in Table 2. In addition, the averaged DVHs based on VM or MM for the 20 patients in whom the plan was recalculated on the registered ConfCT for the CTV and normal liver are shown in Fig. 5; in addition, the dose distributions on the ConfCT using VM and MM in three cases (patients 5, 9, and 18) are shown in Fig. 6.

\subsection{Relationship between the change of WEPL and fiducial marker movement}

The absolute value of the change of the WEPL was measured on the PlanCT and ConfCT. The average and standard deviation was

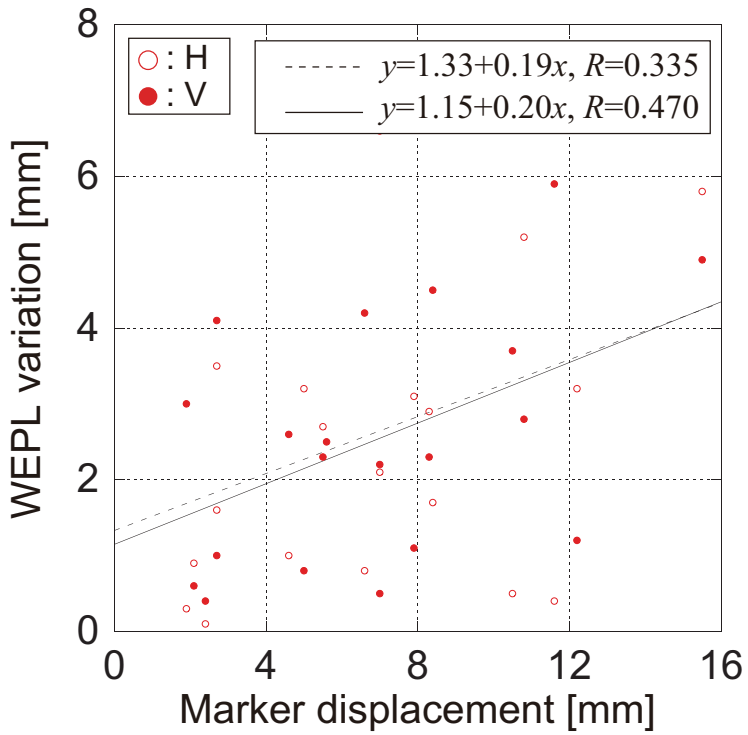

Fig. 7. The relationship between WEPL variation and marker displacement. The vertical axis shows the WEPL variation ( $\mathrm{mm}$ ), and the horizontal axis shows the marker displacement $(\mathrm{mm})$. The red circle indicates the vertical direction, and the white circle indicates the horizontal direction. The black line indicates linear approximation for vertical direction. The dashed line indicates linear approximation for horizontal direction.

$2.63 \pm 2.12 \mathrm{~mm}$ for the lateral beam, and $2.53 \pm 1.61 \mathrm{~mm}$ for the anterior beam. The relationship between the change of WEPL and the absolute of fiducial marker movement is shown in Fig. 7. The gradient of the approximate curve was 0.19 and 0.20 , and correlation coefficient was 0.335 and 0.470 for the lateral and anterior beams, respectively.

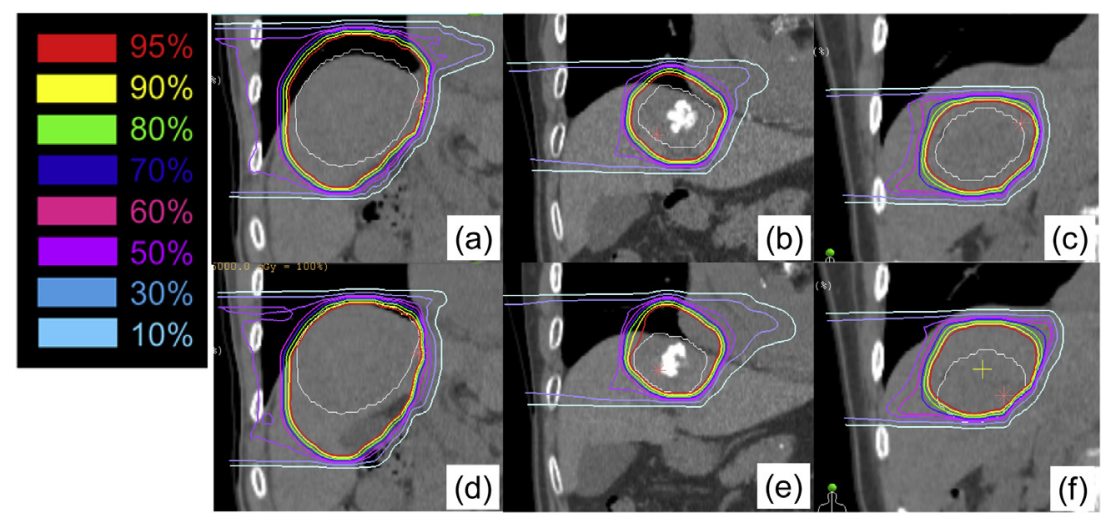

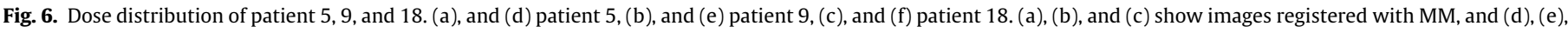
and (f) show images registered with VM. All images are coronal. The CTV is outlined in white. The dose distribution is displayed on a graduated scale from $10 \%$ to $95 \%$. 


\section{Discussion}

\subsection{Comparison of VM and MM}

If $M_{x, y, z}$ was less than $T M_{x, y, z}$ it can be expected that a sufficient dose could be delivered to the target using VM. However, Fig. 4 shows that even if $\Delta D$ was less than 0 , there were some cases when a sufficient dose could not be delivered to the target. This lack of coverage may have resulted from changes in the tumor position within $T M_{x, y, z}$, which influences the WEPL and subsequent variations in inter-fractional errors due to changes in patient anatomy. Based on the linear approximation of Fig. 4, VM seemed to be effective only when $\Delta D$ was less than $-1.8 \mathrm{~mm}$ to achieve sufficient dose to the target. When $\Delta D$ was over $-1.8 \mathrm{~mm}$, alignment with VM may not achieve sufficient dose to the target.

For comparison, the $\mathrm{D}_{98}$ for all patients aligned with MM were clinically acceptable. If the tumor position matched the planned irradiation position with MM, the WEPL reproducibility to the target between the PlanCT and the ConfCT should allow for sufficient dose to the target. From Fig. 7, even if the $M_{x, y, z}$ was large, the correlated WEPL change was low. Additionally, the magnitude of the WEPL change on the isocenter with MM ranged from 0.1 to $7 \mathrm{~mm}$, and in many cases was similar to, or less than $T M_{x, y, z}$. For these cases, it was concluded that a sufficient dose was given to the target.

However, the WEPL changes for patients $4,5,6,8,9,15,17$, and 18 were over the $T M_{x, y, z}\left(2.9 \mathrm{~mm}\right.$ maximum). The $\mathrm{D}_{98}$ determined with MM were less than those of the PlanCT for those patients. Nevertheless, these cases were still clinically acceptable. Meanwhile, the $D_{98}$ for the horizontal beam for patient 19 was $90 \%$ of the prescription dose, and was not clinically acceptable. This was because the magnitude of the change in WEPL was $4 \mathrm{~mm}$ larger than $T M_{x, y, z}$. However, the combined dose distribution from both beams for patient 19 was clinically acceptable.

Table 2 shows that the $D_{98}, V_{80}, V_{90}$, and $V_{95}$ for $M M$ aligned cases were significantly higher than those of VM. Meanwhile, compared with the PlanCT, the $\mathrm{V}_{95}$ for MM were significantly lower. The other indexes showed no significant differences. Although the $\mathrm{V}_{95}$ of MM were significantly different than those of the PlanCT, the dose distributions of MM were unlikely to be a clinical problem because they satisfied the acceptance tolerance. Moreover, $\mathrm{V}_{20}$ for VM and MM have no significant differences, and were clinically safe as shown in Fig. 5. There were three patterns regarding the positional relationship between the liver and the irradiation field. In the first pattern, the tumor was in the upper position of the liver, and moved in the cranial direction (Fig. 6(a), (d)). Thus, the $V_{20}$ for $\mathrm{VM}$ increased relative to the $\mathrm{V}_{20}$ for $\mathrm{MM}$. In the second pattern, the tumor was also in the upper position of the liver, but had moved in the caudal direction (Fig. 6(b), (e)). Thus, the $\mathrm{V}_{20}$ for VM decreased relative to the $\mathrm{V}_{20}$ for $\mathrm{MM}$. In the third pattern, the tumor was positioned in the center of the liver, and did not move appreciably in any direction (Fig. 6(c), (f)). Thus, the $\mathrm{V}_{20}$ for VM did not change relative to the $\mathrm{V}_{20}$ for the $\mathrm{MM}$; consequently, this lack of movement might have been responsible for there being no significant difference between the $\mathrm{V}_{20}$ for VM and MM.

Finally, if an OAR such as the intestine is near the target, the dose distribution in the OAR should be considered. However, this study did not include such cases.

From the above results, the MM method, which does not depend on marker movement was robust enough to deliver an acceptable prescription dose to the target compared with the VM method which has an observed threshold level of $\Delta D<-1.8 \mathrm{~mm}$.

\subsection{Limitations}

There were three limitations of our study regarding the influence of a $\theta_{z}$ error on the dose distribution and the reproducibility of internal physiology during therapy. First, in this study, it was found that the dose distributions of the MM cases were clinically acceptable for all patients. If the MM method is alternated with the tumor matching method, it should be based on highpositional reproducibility between MM and the tumor matching. Seppenwoolde et al. reported that the reproducibility of the difference from the marker to the tumor was $1.6 \pm 1.9 \mathrm{~mm}$ if the threedimensional lengths between the fiducial marker and the tumor center were $6 \mathrm{~cm}$ [17]. In this study, the three-dimensional lengths between the fiducial marker and the tumor center were smaller than $5 \mathrm{~cm}$. Accordingly, it is considered that the positional reproducibility between the fiducial marker and the tumor was high, and any positional error did not substantially effect target dose distribution. However, if the fiducial marker position is far from the tumor position (as observed with some patients in this study), it should be carefully determined if the $D_{98}$ can be meet clinical acceptance criteria.

Second, the CT images acquired in the simulation room were limited to a $\theta_{z}=0^{\circ}$. Thus, even if a $\theta_{z}$ error was induced during position matching with X-rays, the $\theta_{z}$ had to be returned to $0^{\circ}$. Additionally, the registrations with VM and MM were only simulated regarding translational degrees of freedom without rotations, although the registrations with VM and MM for the treatment were performed using six degrees of freedom with translations and rotations. Therefore, the position of the patient on the ConfCT may differ from that of the treatment. However, errors in rotations are typically small (less than $1.0^{\circ}$ ) and have minimal impact on the resulting dose distribution.

Third, this verification was performed on the ConfCT, which was acquired prior to the first treatment day. In addition, although our results show the MM method to be robust, the patient sample size was limited 20 and a larger number of patients should be studied in the future. Finally, our study used a CT scanner in a separate simulation room, which may have induced small positional changes between the scanned position and treatment position. An in-room CT should would provide a more accurate method of verification such that the dose distribution could be assessed on the patient closer to the time of treatment, and also allow for adaptive therapy to be performed.

\section{Conclusion}

In this study, the MM and VM methods were compared and evaluated for effectiveness with CIRT of hepatic cancers. Each method had similar dose coverage for normal liver tissue. However, the MM method was better than the VM method for achieving dose coverage of the CTV, The VM method, below a threshold level $(\Delta D<-1.8 \mathrm{~mm}$ ) was able to meet clinical acceptance criteria for $D_{98}$. Therefore, it was found that the MM, which does not depend on $M_{x, y, z}$, was more capable to reliably deliver the prescribed dose to the target.

\section{Conflict of interest statement}

The authors have no conflict of interest to declare.

\section{Presentation at a conference}

The content of the paper has not been presented at any previous conferences. 


\section{Funding}

This work was supported by Grant-in-Aid for Young Scientists (B) Number 26860396 of Japan Society for the Promotion of Science (JSPS).

\section{Acknowledgments}

The authors would like to thank the radiology technicians, medical doctors, and medical physicists at GHMC for their valuable insight.

\section{References}

[1] Kamada T, Tsujii H, Blakely EA, Debus J, De Neve W, Durante M, et al. Carbon ion radiotherapy in Japan: an assessment of 20 years of clinical experience. Lancet Oncol 2015;16:e93-e100.

[2] Ogino T. Clinical evidence of particle beam therapy (proton). Int J Clin Oncol 2012;17:79-84.

[3] Anderle K, Stroom J, Pimentel N, Greco C, Durante M, Graeff C. In silico comparison of photons versus carbon ions in single fraction therapy of lung cancer. Phys Med 2016;32:1118-23.

[4] Kubota Y, Tashiro M, Shinohara A, Abe S, Souda S, Okada R, et al. Development of an automatic evaluation method for patient positioning error. J Appl Clin Med Phys 2015;16:100-11.

[5] Ohno T. Particle radiotherapy with carbon ion beams. EPMA J 2013;4(9):1-7.

[6] Worm ES, Høyer M, Fledelius W, Hansen AT, Poulsen PR. Variations in magnitude and directionality of respiratory target motion throughout full treatment courses of stereotactic body radiotherapy for tumors in the liver. Acta Oncol 2013;52:1437-44.

[7] Trofimov A, Nguyen PL, Efstathiou JA, Wang Y, Lu HM, Engelsman M, et al. Interfractional variations in the setup of pelvic bony anatomy and soft tissue, and their implications on the delivery of proton therapy for localized prostate cancer. Int J Radiat Oncol Biol Phys 2011:80:928-37.

[8] Méndez Romero A, Zinkstok RT, Wunderink W, van Os RM, Joosten H, Seppenwoolde Y, et al. Stereotactic body radiation therapy for liver tumors: impact of daily setup corrections and day-to-day anatomic variations on dose in target and organs at risk. Int J Radiat Oncol Biol Phys 2009;75:1201-8.

[9] Worm ES, Høyer M, Fledelius W, Poulsen PR. Three-dimensional, timeresolved, intrafraction motion monitoring throughout stereotactic liver radiation therapy on a conventional linear accelerator. Int J Radiat Oncol Biol Phys 2013;86:190-7.

[10] Poulsen PR, Worm ES, Petersen JBB, Grau C, Fledelius W, Høyer M. Kilovoltage intrafraction motion monitoring and target dose reconstruction for stereotactic volumetric modulated arc therapy of tumors in the liver. Radiother Oncol 2014;111:424-30.

[11] Kulkarni NM, Hong TS, Kambadakone A, Arellano RS. CT-guided implantation of intrahepatic fiducial markers for proton beam therapy of liver lesions: assessment of success rate and complications. Am J Roentgenol 2015;204: W207-13.

[12] Trumm CG, Häussler SM, Muacevic A, Stahl R, Stintzing S, Paprottka PM, et al. CT fluoroscopy-guided percutaneous fiducial marker placement for CyberKnife stereotactic radiosurgery: technical results and complications in 222 consecutive procedures. J Vasc Interventional Radiol 2014:25:760-8.

[13] Boulin M, Delhom E, Pierredon-Foulongne MA, Cercueil JP, Guiu B. Transarterial chemoembolization for hepatocellular carcinoma: an old method, now flavor of the day. Diagn Interventional Imaging 2015;96:607-15.

[14] Wunderink W, Méndez Romero A, de Kruijf W, de Boer H, Levendag P, Heijmen B. Reduction of respiratory liver tumor motion by abdominal compression in stereotactic body frame, analyzed by tracking fiducial markers implanted in liver. Int J Radiat Oncol Biol Phys 2008;71:907-15.

[15] Mukumoto N, Nakamura M, Sawada A, Suzuki Y, Takahashi K, Miyabe Y, et al Accuracy verification of infrared marker-based dynamic tumor-tracking irradiation using the gimbaled X-ray head of the Vero4DRT (MHI-TM2000) Med Phys 2013;40:041706.

[16] Seppenwoolde Y, Wunderink W, Wunderink-van Veen SR, Storchi P, Méndez Romero A, Heijmen BJ. Treatment precision of image-guided liver SBRT using implanted fiducial markers depends on marker-tumour distance. Phys Med Biol 2011;56:5445-68.

[17] van Der Horst A, Wognum S, Dávila Fajardo R, de Jong R, van Hooft JE, Fockens $P$, et al. Interfractional position variation of pancreatic tumors quantified using intratumoral fiducial markers and daily cone beam computed tomography. Int J Radiat Oncol Biol Phys 2013;87:202-8.

[18] Wunderink W, Méndez Romero A, Seppenwoolde Y, de Boer H, Levendag P, Heijmen B. Potentials and limitations of guiding liver stereotactic body radiation therapy set-up on liver-implanted fiducial markers. Int J Radiat Oncol Biol Phys 2010;77:1573-83.

[19] Vedam SS, Keall PJ, Kini VR, Mohan R. Determining parameters for respirationgated radiotherapy. Med Phys 2001;28:2139-46.

[20] Minohara S, Kanai T, Endo M, Noda K, Kanazawa M. Respiratory gated irradiation system for heavy-ion radiotherapy. Int J Radiat Oncol Biol Phys 2000;47:1097-103.

[21] Tashiro M, Ishii T, Koya J, Okada R, Kurosawa Y, Arai K, et al. Technica approach to individualized respiratory-gated carbon-ion therapy for mobile organs. Radiol Phys Technol 2013;6:356-66.

[22] Shirato H, Shimizu S, Kitamura K, Nishioka T, Kagei K, Hashimoto S, et al. Fourdimensional treatment planning and fluoroscopic real-time tumor tracking radiotherapy for moving tumor. Int J Radiat Oncol Biol Phys 2000;48:435-42.

[23] Mori S, Kumagai M, Karube M, Yamamoto N. Dosimetric impact of 4DCT artifact in carbon-ion scanning beam treatment: worst case analysis in lung and liver treatments. Phys Med 2016;32:787-94.

24] Kanematsu N, Akagi T, Takatani Y, Yonai S, Sakamoto $\mathrm{H}$, Yamashita $\mathrm{H}$. Extended collimator model for pencil-beam dose calculation in proton radiotherapy. Phys Med Biol 2006;51:4807-17.

[25] Kanematsu N, Torikoshi M, Mizota M, Kanai T. Secondary range shifting with range compensator for reduction of beam data library in heavy-ion radiotherapy. Med Phys 2007;34:1907-10.

[26] Kanematsu N, Yonai S, Ishizaki A. The grid-dose-spreading algorithm for dose distribution calculation in heavy charged particle radiotherapy. Med Phys 2008;35:602-7.

[27] Kanematsu N. Dose calculation algorithm of fast fine-heterogeneity correction for heavy charged particle radiotherapy. Phys Med 2011;27:97-102.

[28] Kanai T, Endo M, Minohara S, Miyahara N, Koyama-ito H, Tomura H, et al Biophysical characteristics of HIMAC clinical irradiation system for heavy-ion radiation therapy. Int J Radiat Oncol Biol Phys 1999;44(1):201-10.

[29] Renner TR, Chu WT. Wobbler facility for biomedical experiments. Med Phys 1987; 14:825-34.

[30] Kanda Y. Investigation of the freely available easy-to-use software "EZR" for medical statistics. Bone Marrow Transplant 2013;48:452-8. 